\title{
LTE Antenna Port Number Detection Algorithm Based on Kalman Autoregression Filtering
}

\author{
Pengchun Jiang, Zengshan Tian, Mu Zhou, Zhihao Li \\ School of Communication and Information Engineering, Chongqing University of \\ Posts and Telecommunications, Chongqing 400065, China \\ 13368124190@163.com, tianzs@cqupt. edu.cn, zhoumu@cqupt. edu.cn, 245719347557@ \\ qq. com,
}

\begin{abstract}
In the LTE system, the traditional detection of number of antenna ports uses blind detection to decode the 1,2 , and 4 port numbers respectively until the system message in physical broadcast channel successfully passes the cyclic redundancy check. This method generates a large amount of computational redundancy and delay. In response to this problem, this paper proposes an improved Kalman autoregressive antenna port number detection algorithm. This algorithm obtains channel state information by extracting the cell reference signals corresponding to different antenna ports, performs Kalman autoregression on the phase information of channel states, and consequently determine the number of antenna ports. Theoretical analysis and simulation results show that the algorithm has low complexity, small delay and a high accuracy rate even when the residual frequency offset is relatively large.
\end{abstract}

Key words: LTE system, antenna port number detection, channel state information, kalman autoregression

\section{Introduction}

Long Term Evolution (LTE), compared with the previous generation communication system, it has the significant increase of data transmission rate and system capacity is largely due to the adoption of Multiple Input Multiple Output (MIMO) technology [1]. The transmitting end can choose to use 1, 2, or 4 antennas to send Physical Broadcast Channel (PBCH) system messages. The User Equipment (UE) performs the PBCH message decoding after the cell search and downlink synchronization operations are conducted [2]. The PBCH can be successfully decoded only if the correct antenna port number configuration information is obtained.

The traditional antenna port number blind detection algorithm repeats P$\mathrm{BCH}$ decoding by traversing all possible antenna ports [3]. This method requires up to three complete decoding, so the calculation amount is large and the delay is high. In [4], the authors propose a power detection algorithm that estimates the signal-to-noise ratio of possible antenna ports using the antenna reference signal and secondary synchronization code power of each antenna port, and then 
uses the signal-to-noise ratio to perform threshold decision to obtain the number of antenna ports. However, when the channel signal-to-noise ratio is relatively poor, the performance of the algorithm drops sharply. The correlation detection algorithm proposed by the authors in [5] is based on the repeatable correlation properties of cell reference signals of different antenna ports and extract the data related to the reference signal position corresponding to the antenna port, then determining whether to use the antenna port to transmit data according to the size of the correlation value.

Based on the above algorithm, this paper proposes an antenna port estimation algorithm with lower complexity and stronger frequency offset resistance, namely Kalman autoregressive antenna port number detection algorithm. This algorithm is based on the Channel State Information (CSI) which is fitted by the Kalman filtering algorithm. The phase average difference between the phase scatter plot and the fitted graph is compared with a set threshold, and the number of antenna ports used by the transmitting end is determined. Theoretical analysis and simulation results show that the proposed algorithm can save up to $60 \%$ of time cost compared with the traditional blind detection algorithms, and meanwhile compared with other optimized threshold decision algorithms, the proposed one is able to improve detection success rate by about $10 \%$ with the same Signal to Noise Ratio (SNR) and featured with better anti-frequency deviation performance.

The structure of this paper is as follows: Section 2 introduces the CSI calculation method. The proposed algorithm is presented in section 3 , section 4 presents simulation results and a conclusion is provided in section 5 .

\section{CRS extracts channel status information}

After the cell search and the downlink synchronization operation are completed, the UE extracts the information corresponding to the reference signal [6] in the time-frequency resource map according to the synchronized frame start position, and compares the local signals and actual received reference signals to complete part of the channel estimation for transmitting the data. It is estimated that Least Squares(LS) is then used to estimate the complete CSI.

The CRS performs corresponding mapping on the time-frequency resource map according to the antenna port number used for transmitting data. Fig. 1 shows the mapping position of the CRS when transmitting data using antenna ports $0,1,2$, and 3 respectively [7]. When the antenna port is $p=0 / 1$, the CRS is located on the 1st or the 3rd OFDM symbol of each slot. when the antenna port is $\mathrm{p}=2 / 3$, the CRS is located in the 2 nd OFDM symbol of each slot.

Channel estimation by comparing the locally generated reference signal with the received reference signal [8]. We assume that the frequency domain information of transmitting end which are not undergone OFDM modulation is $S(\mathrm{j} \omega)$ and the baseband signal information is $s(t)$. After $s(t)$ transmitted by the transmitting end, and receiving by the receiving end, the received signal $r(t)$ is obtained as 

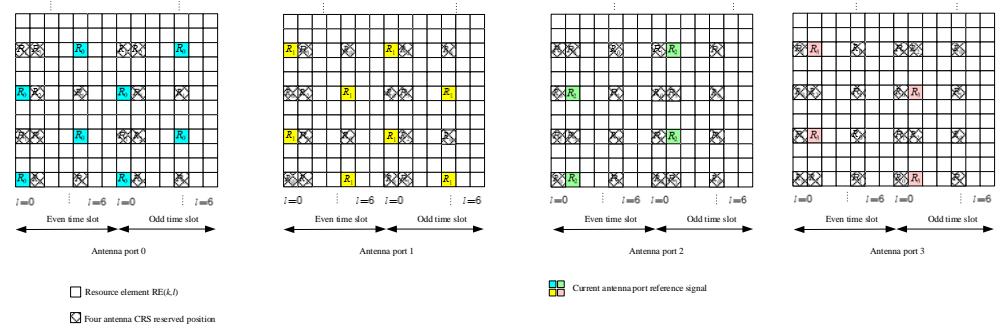

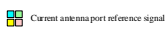

Fig. 1. Resource map of CRS at different antenna ports.

$$
r(t)=A \cdot s(t+\Delta t) e^{\mathrm{j} \Delta \omega t}+n
$$

where $\mathrm{A}$ indicates the amplitude gain during channel transmission, $\Delta t$ is the transmission delay, $\Delta \omega$ is frequency offset, is gaussian white noise, in order to simplify the calculation, $\mathrm{n}$ is ignored. Received signal $r(t)$ through OFDM demodulation [9] can obtain $R(\mathrm{j} \omega)$.

$$
\mathrm{R}(\mathrm{j} \omega)=A \cdot e^{\mathrm{j}(\omega-\Delta \omega) \Delta t} S[\mathrm{j}(\omega-\Delta \omega)]
$$

then get the channel estimation result:

$$
H(\mathrm{j} \omega)=R(\mathrm{j} \omega) / S(\mathrm{j} \omega)=\frac{A \cdot e^{\mathrm{j}(\omega-\Delta \omega) \Delta t} S[\mathrm{j}(\omega-\Delta \omega)]}{S(\mathrm{j} \omega)}
$$

It can be known from equation (10) that the delay of the transmission signal $\Delta t$ in the frequency domain causes a change in the frequency $\omega$ and causes the phase value of the channel estimation to rotate, and the rotation conforms to a phase linear change. Therefore, if the CRS information corresponding to the correct antenna port is extracted, and the complete channel state information [10] is obtained by the least squares method, the phase has a linear change characteristic. Conversely, the channel estimation phase values of successive sampling points will exhibit an irregularly distributed state.

\section{Kalman autoregressive antenna port number detection algorithm}

In order to judge whether the phase of the channel estimation value obtained by the CRS corresponding to different antenna ports satisfies the linear variation law, this paper proposes a method of using the smoothing filter characteristic of the Kalman autoregressive algorithm to perform discrete phase fitting on the received signal channel estimation value. 


\subsection{Kalman autoregressive algorithm}

The Kalman filter algorithm is an optimized autoregressive data processing algorithm consisting of two parts: prediction and update. The role of the prediction part is to estimate the current epoch based on the previous epoch given the initial value. the effect of the update part is to use the actual observation as the prediction part correction [11].

The process model of the algorithm introduces a discrete control system, as follows:

$$
X(k)=A \cdot X(k-1)+B \cdot U(k)+W(k)
$$

The measurement model can be expressed as:

$$
Z(k)=H \cdot X(k)+V(k)
$$

Where $X(k)$ indicates the system state at time $\mathrm{k}, U(k)$ indicates the amount of control of the system at time $\mathrm{k}$. and are system parameters and is the parameter of the measurement system. $Z(k)$ indicates the measured value at time $\mathrm{k}$, $W(k)$ and $V(k)$ representing the noise of the process model and the measurement model, respectively. They are usually assumed to be Gaussian white noise, and their covariances are represented by $\mathrm{Q}$ and $\mathrm{R}$, respectively.

The role of the process model is to predict the state of the system at the next moment [12]. Assuming that the current system state is $\mathrm{k}$, the current state can be predicted by the previous state of the system, as follows:

$$
X(k \mid k-1)=A \cdot X(k-1 \mid k-1)+B \cdot U(k)
$$

where $X(k-1 \mid k-1)$ is the optimal result at the state, $X(k \mid k-1)$ is the result predicted based on the previous state. After the system results are updated, the covariance of $X(k \mid k-1)$ is represented by $P$.

$$
P(k \mid k-1)=A \cdot P(k-1 \mid k-1) \cdot A^{\prime}+Q
$$

Equations (13) and (14) are the first two of the Kalman filter formulas and belong to the prediction part of the system.

After obtaining the prediction result of the current state, it is also necessary to collect the measurement value of the current state $X(k \mid k)$, and the two obtain the optimal estimation value of the current state by using equation (8).

$$
X(k \mid k)=X(k \mid k-1)+K g(k)(Z(k)-H \cdot X(k \mid k-1))
$$

where $K g$ is the Kalman gain.

$$
K g(k)=P(k \mid k-1) \cdot H^{\prime} /\left(H \cdot P(k \mid k-1) \cdot H^{\prime}+R\right)
$$

Then update the covariance of $X(k \mid k)$ in the k state,

$$
P(k \mid k)=(l-K g(k) \cdot H) \cdot P(k \mid k-1)
$$


Where $l$ is the full 1 matrix. For the next state of the system, $P(k \mid k)$ updates as $P(k-1 \mid k-1)$ in equation (16), in turn, the autoregressive operation of the algorithm is implemented.

\subsection{Kalman autoregressive implementation of antenna port number detection}

For the receiving end, correctly decoding the $\mathrm{PBCH}$ information requires obtaining a channel estimation of the transmitted signal and a mapped position of the $\mathrm{PBCH}$ signal in the time-frequency resource map. The received signals $Y(k)$ are respectively extracted according to these positions, according to Equation (10) is divided by the local CRS to obtain the channel estimation, and the complete channel estimation $\hat{H}_{\mathrm{LS}}$ is obtained by the least squares algorithm:

$$
\hat{H}_{L S}=F_{L}\left(F_{p}^{\mathrm{H}} F_{p}\right)^{-1} F_{p}^{\mathrm{H}} \hat{H}_{p}
$$

where $p$ is the reference signal, $F_{p}$ is a PL order matrix, the row corresponding to the reference symbol position selection and the first L column of the NN Discrete Fourier Transform (DFT) matrix. $F_{L}$ is an $\mathrm{N}$ L order matrix, which is obtained by the first $\mathrm{L}$ column of the DFT matrix. $\hat{H}_{p}$ is a complex-valued cyclic white noise vector with a $\mathrm{P} 1$ order mean value of zero.

In order to determine whether the antenna port is used, this paper proposes a method of autoregressive fitting of phase discrete points of $\hat{H}$ using Kalman filter. When the correct antenna port is detecting, the received signal phase discrete point map and the phase fitting line can be highly matched. Conversely, because of the smoothing characteristics of the Kalman algorithm, the fitted line after autoregression reduces the amplitude of the sample phase fluctuation. The fitting effect is shown in Fig. 2. The blue line represents the phase fitting line of the Kalman filter algorithm, the red dot represents the sampling point phase of the channel estimation value $\mathrm{A}$, and the left side is the case after the channel estimation using the CRS of the correct port. The fitted line highly fits the discrete point. the right side is the case after the channel estimation using the CRS of the wrong port, and the phase difference between the fitted line after the auto-regression and the channel estimation is large.
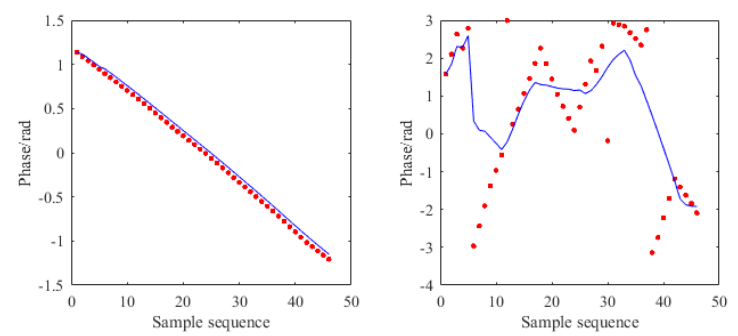

Fig. 2. Correct/Error Port Kalman Filter Phase Fitting Diagram. 
The Kalman filter algorithm is used to perform the autoregressive fitting of the phase points. First, the initial system state $X(1)$ and the initial covariance $P(1)$ are assigned. The covariance of the process noise and the measurement noise is represented by $\mathrm{Q}$ and $\mathrm{R}$, and the equation (6) is obtained under the system. The predicted value $X(k \mid k-1)$ of a state. The equation (8) corrects the filtering result $\hat{H}$ by using the value in the channel condition information matrix $X(k \mid k)$ calculated by the least squares method as a measured value. Then calculate the average value of the absolute phase difference between the sample sequence $\mathrm{k}$ and the discrete point $\mathrm{k}$ on the autoregressive line after filtering.

$$
\bar{\varepsilon}=\frac{\sum_{k=1}^{N}\left|\varepsilon_{k}\right|}{N}=\frac{\sum_{k=1}^{N}|X(k \mid k)-Y(k)|}{N}
$$

$Y(k)$ represents the phase value of the channel estimation of the sampling point $k$. In this paper, it is determined whether the current antenna port is configured with CRS by comparing the error average value $\bar{\varepsilon}$ of different antenna ports with the preset threshold $\gamma$. The four antenna ports are sequentially determined in the order given in Fig. 3, and finally the number of antenna ports used by the transmitting end is determined. After the simulation test, the threshold can usually be set between 0.3 0.5. This article presets $\gamma=0.4$. The specific processing steps of the entire decision process are as follows:

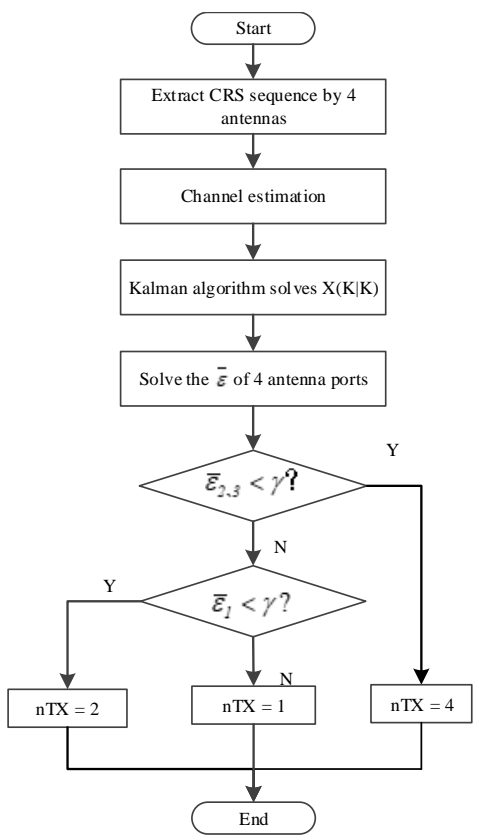

Fig. 3. Flow chart of the number of antenna ports based on Kalman filtering. 
First, the absolute average value of error of the antenna ports 2 and 3 is determined. If it is less than the threshold $\gamma$, the number of antenna ports is determined to be four. Otherwise, the absolute average value of error of the antenna port 1 is determined, and if it is less than the threshold $\gamma$, it is determined that the number of antenna ports is two, and the number of antenna ports is determined to be one.

\section{Performance simulation analysis}

This section first verifies the correctness of the phase fitting results of the Kalman autoregressive antenna port number detection algorithm at different antenna ports, and secondly compares the detection performance of several existing algorithms under different SNR and frequency offset, and finally compares them on the same platform. The time consuming of each algorithm.

\subsection{Verification of phase fitting effect of different antenna port numbers}

This section simulates the generation of LTE downlink signals according to specific signal parameters. The reference signals of the corresponding positions of the four antenna ports are respectively extracted to obtain possible channel state information, and the number of antenna ports is determined according to the flow of Fig. 3 which using the Kalman autoregressive algorithm. Fig. 4 to Fig. 6 are constellation diagrams and phase diagrams after channel estimation of CRS of antenna ports $0,1,2$, and 3 are extracted at the receiving end when the transmitting end transmits data using 1, 2, and 4 antennas, respectively.

When channel estimation is performed using the correct antenna port, the phase value of the channel estimation will form a relatively concentrated arc under the action of frequency offset and phase offset, as shown in the port0 subgraph of Fig. 4 (a). Channel estimation with reference signals causes the points on the constellation to be relatively dispersed. Therefore, this paper proposes a Kalman autoregressive algorithm to detect whether the points on the constellation map are relatively concentrated or scattered. Fig. 4(b) is a 45-point linear matching result of a single antenna port of the Kalman filter algorithm. The red discrete point represents the phase value of the received signal, and the blue line represents the fitted line of the discrete point phase after Kalman filtering. The effect of the phase is that the phase dispersion point can be well matched when the phase changes regularly. And when the phase fluctuation is large, the phase difference between the fitted line and the discrete point after smoothing is larger. According to this characteristic, the absolute value of the absolute phase difference of the same point and the fitted regression line are compared with the preset threshold value, thereby obtaining the correct number of antenna ports. After the simulation test, the threshold $\gamma$ is generally in the range of 0.4 to 0.6 . In this paper, the value of $\gamma$ is 0.5 . 

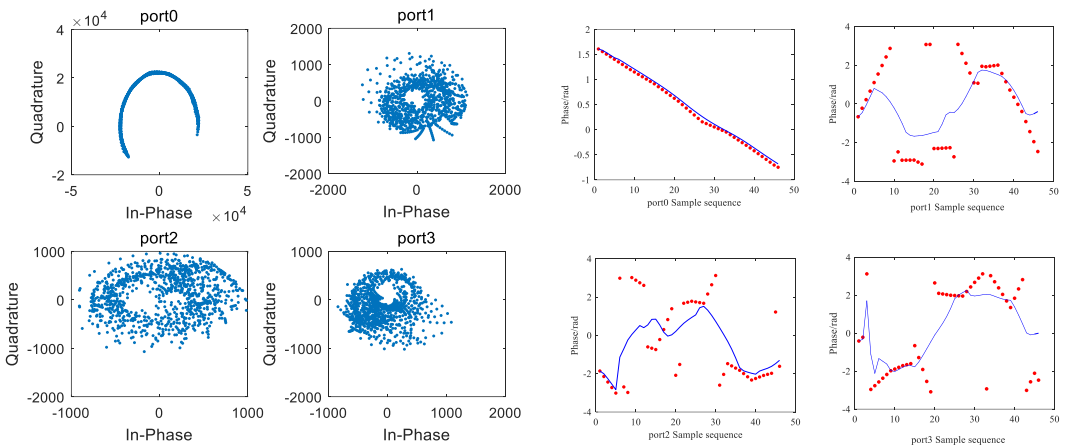

(a) constellation diagram

(b) Kalman phase fitting diagram

Fig. 4. Single antenna constellation diagram and phase fit diagram.
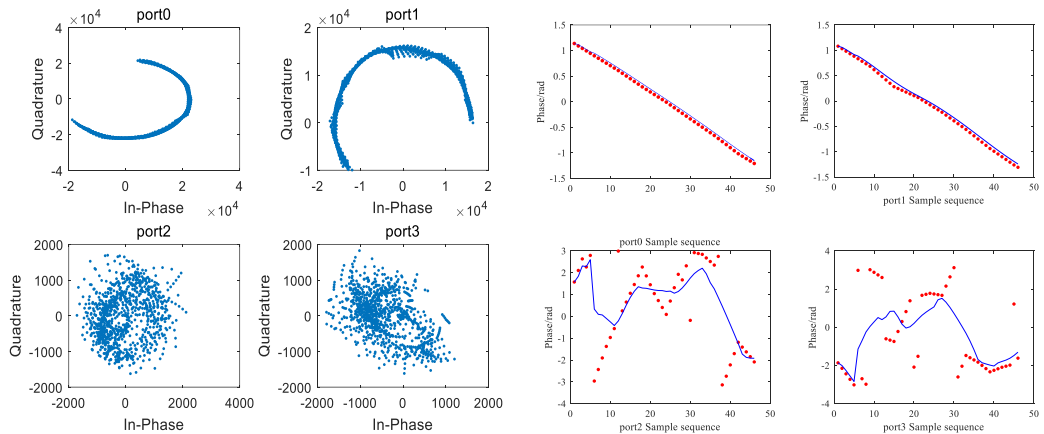

(a) constellation diagram

(b) Kalman phase fitting diagram

Fig. 5. Two antenna constellation and phase fitting diagram.
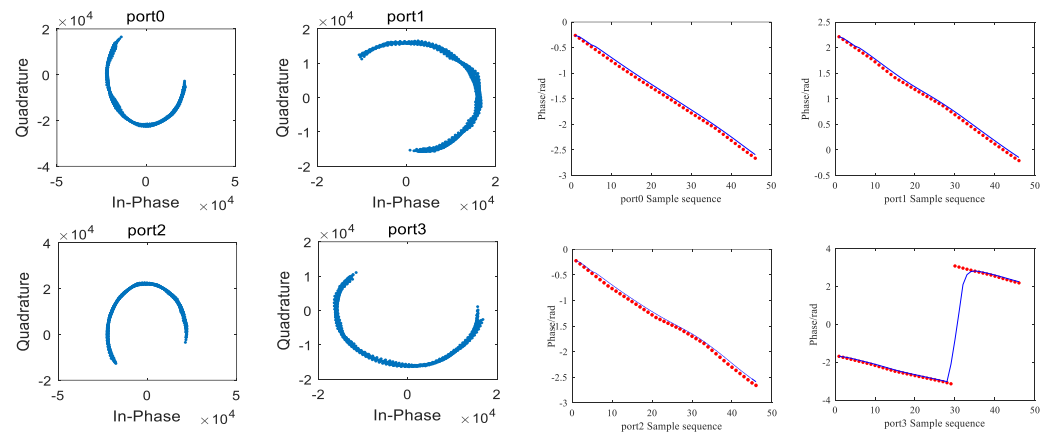

(a) constellation diagram

(b) Kalman phase fitting diagram

Fig. 6. Four antenna constellation diagram and phase fitting diagram. 
According to the measured data sent by the two antenna ports, verification can be obtained that $\bar{\varepsilon}_{0}=0.0539, \bar{\varepsilon}_{1}=0.0733, \bar{\varepsilon}_{2}=0.7353, \bar{\varepsilon}_{3}=0.6741$. According to the process of Fig. 5, the threshold decision is made, and the number of antenna ports can be correctly solved as two.

\subsection{Algorithm detection performance comparison}

Although the least squares method obtains a certain frequency offset compensation after channel estimation of the cell-specific reference signal, there is still a case where the frequency offset compensation is large due to inaccurate channel estimation, so the frequency offset residual can better verify the robustness of the detection algorithm. Section 4.1 has verified the availability of the algorith$\mathrm{m}$ when the frequency offset is $100 \mathrm{~Hz}$. This section adds the power detection algorithm and the correlation detection algorithm to the comparison, and adds the frequency offset to $500 \mathrm{~Hz}$. The detailed parameters are as follows: $\rho$ in the power detection algorithm is set to $0.75, T_{1}$ in the correlation detection algorithm is set to 0.65 , and the threshold $\gamma$ of the algorithm is set to 0.5 while 2000 decoding simulation tests are performed. If the $\mathrm{PBCH}$ message can pass the CRC check, it is determined that the antenna port detection is successful. The detection success rate is shown in Fig. 7.

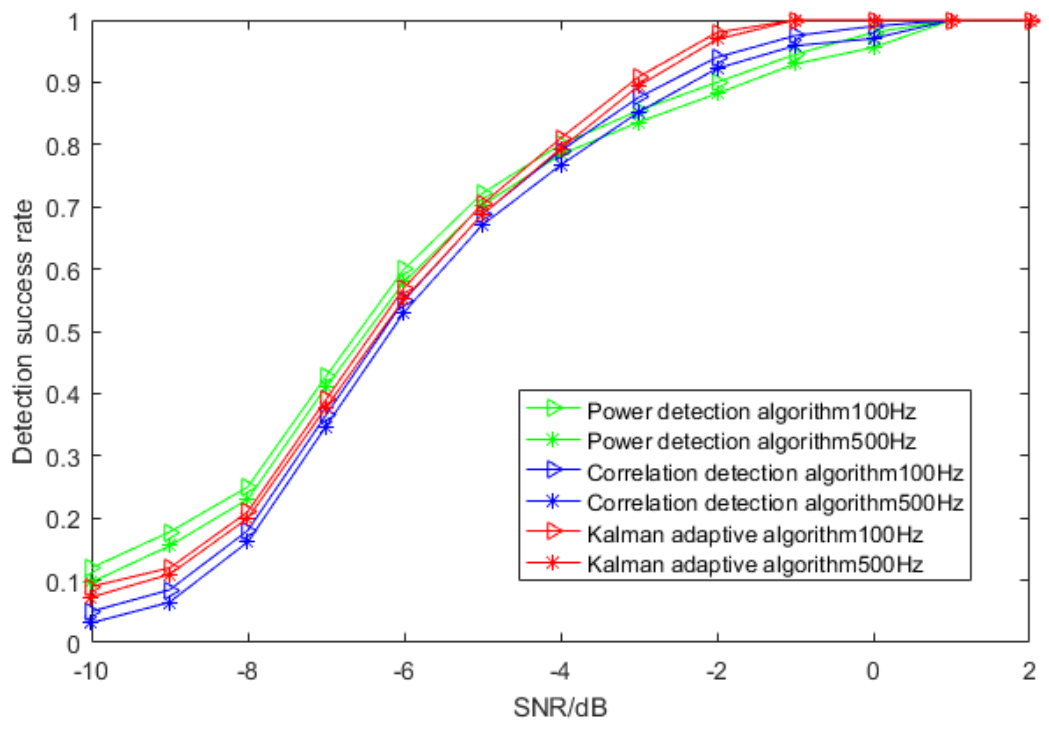

Fig. 7. Comparison of success rates of three detection algorithms under different frequency offsets.

It can be seen from Fig. 7 that the frequency offset always has an influence on the algorithm detection success rate, and the smaller the signal frequency 
offset is, the higher the detection success rate this algorithm has. In the case of low SNR, the power detection algorithm uses blind detection to detect, so the detection success rate is higher than the other two algorithms. When the signal-to-noise ratio condition meets the SNR requirements, its detection success rate is the lowest of above three methods. Taking the case of SNR of $-2 \mathrm{~dB}$ as an example, after the frequency offset is increased from $100 \mathrm{~Hz}$ to $500 \mathrm{~Hz}$, the detection success rate of this algorithm is reduced by $1.1 \%$, and the correlation detection and power detection are reduced by $1.78 \%$ and $1.81 \%$, respectively. Better anti-frequency offset performance, the algorithm is more robust. At the same time, compared with the correlation detection algorithm, in the case of changing the signal-to-noise ratio, the success rate of the algorithm detection is always higher than the former. Therefore, the Kalman autoregressive algorithm in this paper has higher frequency offset, robustness and detection success rate than the other two algorithms.

\subsection{Algorithm detection time comparison}

The algorithm detection time is also an important indicator to measure the performance of the antenna port number detection algorithm. On the basis of ensuring the detection success rate, the lower detection time is beneficial to improve the system response speed and stability. Ideally, the four algorithms mentioned in this paper only perform a complete $\mathrm{PBCH}$ decoding in a single antenna, however the time comparison is not obvious due to the number of antenna ports is unknown. Therefore, in the simulation environment of MATLAB, the four antenna ports with the most obvious time difference are selected for comparison. The time when each algorithm is used for port number detection and $\mathrm{PBCH}$ decoding is shown in Table 3.

Table 1. Comparison of simulation time of different algorithms

\begin{tabular}{lcc}
\hline Detection algorithm & Port detection time $(s)$ & PBCH \\
\hline Traditional algorithm & 0 & 1.006577 \\
\hline Power algorithm & 0.021132 & 0.403956 \\
\hline Correlation algorithm & 0.024402 & 0.401967 \\
\hline Kalman algorithm & 0.027708 & 0.399295 \\
\hline
\end{tabular}

Since the blind detection algorithm directly traverses each possible port number, the port number detection time is 0 , and the decoding of the four antenna ports requires 3 complete $\mathrm{PBCH}$ decoding, resulting in the longest total time. The latter three algorithms only perform decoding once, and the required port number detection time is much lower than that of a complete $\mathrm{PBCH}$ system message decoding time. Therefore, the latter three algorithms take much less time than the blind detection algorithm. The Kalman algorithm in this paper has a slightly higher computational complexity, so the time spent in port number detection is slightly longer than the other two algorithms, but it is much lower than the time required to completely decode the $\mathrm{PBCH}$. 


\section{Conclusion}

In this paper, a Kalman autoregressive antenna port number detection algorithm based on channel estimation phase fitting is proposed. The algorithm extracts the phase of the channel estimation value from the channel estimation result, and uses the channel estimation value through Kalman autoregressive method to determine the number of antenna ports, which solves the problem of the antenna port number decision error caused by the phase mutation of the sampling point of the channel estimation value for fitting. Theoretical and simulation results prove the proposed algorithm has lower complexity performance, better anti-frequency offset performance, robustness and feasibility. The detection of antenna port number directly affects channel estimation and channel decoding, so it is of great significance to study it in detail.

\section{References}

1. LIU K, TAO C, D L, t al. Asymptotic analysis for low-resolution massive MIMO systems with MMSE receiver[J]. China Communications, 2018, 15(9):189-199.

2. M H Nassralla, M M Mansour, and L M A Jalloul. A Low-Complexity Detection Algorithm for the Primary Synchronization Signal in LTE[J]. IEEE Transactions on Vehicular Technology, 2016, 65(10):8751-8757.

3. Z Wang. Method and apparatus for decoding physical broadcast channel in time division duplexing system $[\mathrm{J}]$. International Journal of Machine Tools and Manufacture, 2015, 93:37-48.

4. CHEN F, CHEN B, and WANG D. Design and realization on the number of antennas in LTE system[J]. Journal of Chongqing University of Posts and Telecommunications, 2013.

5. JIANG Q and WEI S. An Improved Antenna Port Number Detection Algorithm for LTE System[J]. Telecommunication Engineering, 2016.

6. YUE G R, HAO T, and LIN Y, et al. SNR Estimation Algorithm Based on Sounding Reference Signal in LTE[J]. Journal of Electronics and Information Technology, 2014.

7. WANG Y Y, HAN F, and YANG Q U, et al. Pilot Based Channel Estimation Algorithm of LTE System over High-Speed Mobile Channels[J]. Journal of Signal Processing, 2018.

8. CHARRADA A and SAMET A. Joint interpolation for LTE downlink channel estimation in very high-mobility environments with support vector machine regression[J]. IET Communications, 2016, 10(17):2435-2444.

9. BOCHKOV G N, GOROKHOV K V, and V A V. Demodulation Algorithms for the OFDM Signals in the Time- and Frequency-Scattering Channels[J]. Radiophysics and0 Quantum Electronics, 2016, 59(1):1-21.

10. WANG Y, ZHANG X, and XIAO L, et al. An improved channel estimation method for LTE downlink system[C]. IEEE Antennas and Propagation. 2016:480-484.

11. QIAO S J, HAN N, and ZHU X W, et al. A Dynamic Trajectory Prediction Algorithm Based on Kalman Filter[J]. Acta Electronica Sinica, 2018.

12. CAEEANO C S, ANGHEL A, and QUINN R A, et al. Kalman filter estimation of plasmaspheric total electron content using GPS[J]. Radio Science, 2016, 44(1):1-14. 